\title{
Crecimiento y producción somática de la concha de abanico (Argopecten purpuratus) en Bahía Independencia, Pisco (Perú) comparados entre eventos El Niño y La Niña
}

\author{
Growth and somatic production of the fan scallop (Argopecten purpuratus) in Independencia Bay, \\ Pisco (Peru) during El Niño and La Niña events \\ Juan Tarazona ${ }^{1,2}$, Roberto Espinoza ${ }^{1}$, Marco Solís ${ }^{1}$ y Wolf Arntz $^{3}$ \\ ${ }^{1}$ Grupo DePSEA Instituto Antonio Raimondi, Laboratorio de Ecología Marina, Facultad de Ciencias Biológicas, Universidad Nacional \\ Mayor de San Marcos. Apartado 1898, Lima 100, Perú \\ ${ }^{2}$ Red sobre Impacto Biológico de los Eventos El Niño (RIBEN), Consejo Nacional de Ciencia y Tecnología. Apartado 1984, Lima 100, Perú \\ ${ }^{3}$ Alfred Wegener Institute for Polar and Marine Research. Am Alten Hafen 26, D-27568, Bremerhaven, Germany \\ respinoza.unmsm@gmail.com
}

\begin{abstract}
Argopecten purpuratus is the most economically important shellfish resource in the central coast of Peru. In the last years, the information about its stock, population structure and temporal changes (recruitment, reproduction, etc.) has increased. However, some aspects of its dynamics during the ENSO periods are still unknown. The present study compares various population parameters of the scallop Argopecten purpuratus between the warm El Niño 1997-98 event and the cold La Niña 1999-2000 event. During El Niño, growth rate was about three times higher than that registered during La Niña, while the population's somatic production and the annual turnover rate $(\mathrm{P} / \mathrm{B})$ were respectively tenfold and twice higher than that observed during La Niña. These results demonstrate positive effects of El Niño on the growth and secondary production of $A$. purpuratus. This knowledge is an important contribution for fishery management system.
\end{abstract}

Key words: Pectinidae, ENSO events, biomass, mortality, $\mathrm{P} / \mathrm{B}$ rate

\section{Introducción}

El efecto del evento El Niño (EN) sobre los invertebrados comerciales de la costa peruana, con especial énfasis en la zona de Pisco, ha sido reportado por varios autores en los últimos años (Wolff 1985, Arntz 1986, Arntz \& Tarazona 1990). Si bien el efecto de EN fue negativo para muchas especies, una
Resumen.- Argopecten purpuratus es el marisco de mayor importancia económica en la costa central del Perú, por su elevado volumen de extracción en la pesca artesanal. En los últimos años se ha incrementado la información sobre su stock, estructura poblacional y cambios en el tiempo (reclutamiento, reproducción, etc.). Sin embargo, aún se desconocen algunos aspectos de su dinámica poblacional ante la presencia de la Oscilación Sureña El Niño (ENOS). En el presente trabajo se hace una comparación de algunos parámetros poblacionales de Argopecten purpuratus durante el evento cálido El Niño 1997-98 y el evento frío La Niña 1999-2000. Durante El Niño, la tasa de crecimiento fue casi tres veces mayor que durante el evento La Niña, la producción somática de la población fue 10 veces mayor, y la tasa de recambio P/B fue el doble que durante La Niña. Estos resultados demuestran los efectos positivos de El Niño sobre el crecimiento y producción secundaria de A. purpuratus. Este conocimiento es un aporte valioso para el sistema de manejo pesquero.

Palabras clave: Pectinidae, ENOS, biomasa, mortalidad, tasa $\mathrm{P} / \mathrm{B}$ apreciable variedad de mariscos como la concha de abanico, los langostinos, el pulpo y las jaibas, aprovecharon el calentamiento $\mathrm{y}$ las mayores concentraciones de oxígeno, característicos de este período, para la proliferación de sus poblaciones, con el consiguiente desarrollo de sus pesquerías. 
La mayor atención de los investigadores se ha concentrado en la concha de abanico Argopecten purpuratus (Lamarck, 1819) extraída en Bahía Independencia, Pisco. Así, en los últimos años se ha incrementado la información sobre su stock, estructura poblacional y distribución (Wolff \& Mendo 2000), así como el conocimiento de la dinámica poblacional, sobre todo crecimiento, reproducción y asentamiento larval (Wolff 1987, Yamashiro \& Mendo 1988, Stotz \& González 1997, Mendo \& Wolff 2002). En esta especie el efecto positivo del evento El Niño se manifiesta principalmente con una reproducción y reclutamiento más intenso que en años "normales", lo que repercute con elevadas capturas en las principales zonas de extracción (Arntz 1984, Wolff 1985). El presente trabajo compara los efectos del evento cálido El Niño (EN) y del evento frío La Niña (LN) sobre el crecimiento y producción somática de $A$. purpuratus en Bahía Independencia, Pisco.

\section{Material y métodos}

\section{Área de estudio y obtención de muestras y datos}

El estudio se desarrolló en la zona de La Pampa en Bahía Independencia, Pisco, Perú. La toma de muestras se realizó mensualmente en una estación fija $\left(14^{\circ} 15^{\prime} \mathrm{S}\right.$; $76^{\circ} 10^{\prime} \mathrm{W}$ ), durante un período cálido El Niño (EN), de junio de 1997 a julio de 1998 (13 muestras); y durante un período frío La Niña (LN), de marzo de 1999 a marzo de 2000 (13 muestras). Los muestreos de la población de $A$. purpuratus se realizaron en faenas de buceo autónomo, en las que se obtuvieron 10 muestras al azar, a profundidades de entre 10 y $14 \mathrm{~m}$, utilizando un marco cuadrado de $1 \mathrm{~m}$ de lado. Los ejemplares fueron pesados y contados para obtener la abundancia y biomasa por área. Adicionalmente se tomaron muestras mediante raleos en las zonas vecinas, cuando la densidad fue baja, y fueron destinadas a las relaciones biométricas. Las anomalías de la temperatura superficial del mar en ambos períodos de estudio se obtuvieron usando un promedio patrón generado a partir de los datos de temperatura superficial del mar en la isla La Vieja, Bahía Independencia, obtenidos por la empresa PROABONOS, durante el período 1978-2000.

\section{Relación talla-peso}

En el laboratorio, las conchas de abanico fueron contadas, medidas y pesadas, previo desprendimiento de los organismos adheridos sobre las valvas. La talla, expresada como altura total $\left(\mathrm{H}_{\mathrm{t}}\right)$ de cada individuo (distancia entre los extremos dorsal y ventral) se midió con un calibre Vernier (precisión: $\pm 0,05 \mathrm{~mm}$ ); y los pesos húmedo visceral (PH) y seco visceral (PSV) se obtuvieron empleando una balanza electrónica, con una precisión de $\pm 0,01 \mathrm{y} \pm 0,0001 \mathrm{~g}$ respectivamente. Las vísceras comprendieron toda la masa visceral incluido el talo y la gónada (peso húmedo libre de conchilla). El peso seco libre de cenizas (PSLC) se obtuvo mediante la calcinación de cenizas de las partes viscerales secas en mufla $\left(600^{\circ} \mathrm{C}, 3 \mathrm{~h}\right)$. Para cada una de las muestras, se determinó la relación PSLC $=\mathrm{a} \mathrm{H}_{\mathrm{t}}^{\mathrm{b}}$ (a $\mathrm{y}$ b son parámetros constantes de la ecuación) mediante el ajuste de regresiones por mínimos cuadrados. Asimismo fue realizada una comparación de las regresiones (ANCOVA) entre ambos períodos (Sokal \& Rohlf 1981).

\section{Parámetros de crecimiento y mortalidad}

Los parámetros de crecimiento y mortalidad para el período EN y LN fueron estimados a partir de la frecuencia de tallas, utilizando el programa computacional FISAT (Gayanilo et al. 1995). Las estimaciones del crecimiento se obtuvieron con la función de crecimiento no oscilante de von Bertalanffy:

$$
H_{t}=H_{\infty}\left(1-e_{0}^{-K(t-t)}\right)
$$

donde:

$\mathrm{H}_{\mathrm{t}}$ es la altura de la valva (talla) a la edad $\mathrm{t}$,

$\mathrm{H}_{\infty}$ es la media asintótica de la altura de la valva,

$\mathrm{K}$ es la constante de crecimiento en años ${ }^{-1}$,

t es la edad en años, $y$

$\mathrm{t}_{0}$ es la edad a la longitud cero.

La mortalidad total $\mathrm{Z}$ (año ${ }^{-1}$ ) fue estimada a partir de los datos de frecuencia de tallas, mediante el método de curva de captura basada en la conversión de tallas (Pauly 1983). Usando los parámetros de crecimiento del modelo von Bertalanffy, la altura de las conchas de abanico agrupadas en intervalos de talla fueron convertidas a edad, usando la siguiente ecuación:

$$
\mathrm{N}_{\mathrm{j}} / \Delta \mathrm{tj}=\mathrm{N}_{0} \mathrm{e}^{-\mathrm{Ztj}}
$$

donde:

$\mathrm{N}_{\mathrm{j}}$ es el número de individuos en el intervalo de talla $\mathrm{j}$. 
$\Delta \mathrm{t}_{\mathrm{j}}$ es el tiempo necesario para crecer a través del intervalo $\mathrm{j}$.

$\mathrm{N}_{0}$ es el número de individuos en el tiempo 0 .

$t_{j}$ es la edad relativa correspondiente a la talla media del intervalo de $\mathrm{j}$.

La edad $t_{j}$ fue calculada utilizando el modelo de crecimiento von Bertalanffy inverso:

$$
\mathrm{t}_{\mathrm{j}}=\operatorname{Ln}\left[1-\mathrm{H}_{\mathrm{j}} / \mathrm{H}_{\infty}\right][1 / \mathrm{K}]+\mathrm{t}_{0}
$$

donde $\mathrm{H}_{\mathrm{j}}$ es la altura de la valva (talla) a la edad $\mathrm{t}_{\mathrm{j}}$.

La mortalidad $\mathrm{Z}$ fue finalmente calculada mediante un análisis de regresión lineal de la siguiente ecuación:

$$
\operatorname{Ln}\left(\mathrm{N}_{\mathrm{j}} / \Delta \mathrm{t}_{\mathrm{j}}\right)=\operatorname{Ln}\left(\mathrm{N}_{0}\right)-\mathrm{Zt}_{\mathrm{j}}
$$

donde $-\mathrm{b}=\mathrm{Z}$

Con fines comparativos, $\mathrm{Z}$ fue también estimado por el método de Jones \& van Zalinge (1981), el cual forma parte del software FISAT.

La mortalidad natural M (año $\left.{ }^{-1}\right)$ fue estimada por el modelo de Hoenig (1983) quien establece:

$$
\operatorname{Ln}(\mathrm{M})=1.44-0.982 \operatorname{Ln}\left(\mathrm{E}_{\text {máx }}\right)
$$

donde $\mathrm{E}_{\text {máx }}$ es la edad máxima en años.

También se estimó $\mathrm{M}$ indirectamente, a partir del cociente $\mathrm{P} / \mathrm{B}$, de acuerdo al método propuesto por Brey $(1995,2001)$, quien utilizó la fórmula:

$\log (\mathrm{P} / \mathrm{B})=1.646+0.995 \log \left(1 / \mathrm{E}_{\text {máx }}\right)-0.634$ $\log \left(\mathrm{M}_{\text {máx }}\right)-293.039(1 / \mathrm{T})$

donde:

$\mathrm{P} / \mathrm{B}=\mathrm{M}$

$\mathrm{E}_{\text {máx }}$ es la edad máxima en años.

$\mathrm{M}_{\text {máx }}$ es la masa promedio máxima expresada en kJ.

$\mathrm{T}$ es la temperatura media anual del agua de mar en grados $\mathrm{K}$.

Para la determinación del valor de $\mathrm{t}_{0}$, que representa el tiempo en que el organismo tiene $0 \mathrm{~mm}$ de altura, se empleó la fórmula sugerida por Pauly (1980):

$$
\log \left(-\mathrm{t}_{0}\right)=-0,3922-0,2752 \log \left(\mathrm{H}_{\infty}\right)-1,038 \log (\mathrm{K})
$$

Para comparar el rendimiento del crecimiento se estimaron dos índices, calculando el valor de $\Phi$ ' (Pauly \& Munro 1984), mediante la ecuación:

$\Phi=\log (\mathrm{k})+2 \log \left(\mathrm{H}_{\infty}\right) ;$

y el de $\psi$ (Brey 1995), mediante la ecuación:

$\Psi=\log \left(\mathrm{M}_{\text {máx }} / \mathrm{E}_{\text {máx }}\right)$

$\mathrm{E}_{\text {máx }}$ es la edad máxima, hallada según $\mathrm{E}_{\text {máx }}=\mathrm{t}_{\mathrm{o}}-$ $1 / \mathrm{K} \operatorname{Ln}\left(1-\mathrm{L}_{\operatorname{máx}} / \mathrm{L}_{\infty}\right)$

\section{Producción y biomasa}

La producción somática anual de la población $\left(\mathrm{P}_{\mathrm{s}}\right)$, expresada en gramos de peso seco libre de cenizas (PSLC), fue calculada usando el método de la tasa de crecimiento específico en peso (Crisp 1984), utilizando los datos de abundancia, las frecuencias de tallas, los parámetros de la función de crecimiento de von Bertalanffy y las relaciones talla-peso:

$$
\mathrm{P}_{\mathrm{s}}=\Sigma \mathrm{N}_{\mathrm{i}} \mathrm{W}_{\mathrm{i}} \mathrm{G}_{\mathrm{i}}\left[\mathrm{g} \mathrm{PSLC} \mathrm{m}^{-2} \mathrm{año}^{-1}\right],
$$

donde:

$\mathrm{N}_{\mathrm{i}}$ es el número promedio de ejemplares $\left(\mathrm{N} \mathrm{m}^{-2}\right)$;

$\mathrm{W}_{\mathrm{i}}$ es el peso corporal promedio en la clase de talla $\mathrm{i}$, expresado en gramos de peso seco libre de cenizas y

$\mathrm{G}_{\mathrm{i}}$ la tasa de crecimiento específico en peso.

$$
\begin{gathered}
\mathrm{G}_{\mathrm{i}}=\mathrm{bK}\left(\left(\mathrm{H}_{\infty} / \mathrm{H}_{\mathrm{i}}\right)-1\right)\left[\mathrm{año}^{-1}\right] \\
\mathrm{P}_{\text {ind }}=\mathrm{W}_{\mathrm{i}} \mathrm{G}_{\mathrm{i}}[\mathrm{g} \text { PSLC } \text { año } \\
\end{gathered}
$$

donde:

b es el exponente de la relación altura-peso,

$\mathrm{H}_{\infty}$ y K son los parámetros de la función de crecimiento de von Bertalanffy y

$\mathrm{H}_{\mathrm{i}}$ es la altura promedio de la valva en la clase de talla i.

La biomasa promedio por clases anual B fue calculada mediante la ecuación:

$$
\mathrm{B}=\Sigma \mathrm{N}_{\mathrm{i}} \mathrm{W}_{\mathrm{i}} \quad\left[\mathrm{g} \text { PSLC m} \mathrm{m}^{-2}\right]
$$


La tasa de renovación anual $\mathrm{P} / \mathrm{B}$ fue calculada a partir de la producción somática anual $\mathrm{P}$ y de la biomasa media anual B.

\section{Resultados}

\section{Anomalías térmicas del agua de mar}

Durante el período cálido de EN 1997-98, la temperatura superficial del mar mostró anomalías positivas, alcanzando una máxima de hasta $+6,89^{\circ} \mathrm{C}$ en enero de 1998; mientras que durante el período frío LN 1999-2000 se registraron negativas, que llegaron hasta $-1,85^{\circ} \mathrm{C}$ en septiembre de 1999 (Fig. 1).

\section{Relación talla-peso}

Durante EN, la relación peso seco libre de cenizas (PSLC) sobre talla obtenida para la población de $A$. purpuratus mostró una pendiente menor $(\mathrm{b}=2,9934)$ que durante LN ( $b=3,4837)$. Así, de acuerdo a las relaciones establecidas, a la talla comercial estándar de $65 \mathrm{~mm}$ le corresponden 1,87 g PSLC durante EN y 4,14 g PSLC durante LN. Ambas curvas se aproximan en las tallas menores, mostrando una intersección a una talla aproximada de 25,5 mm, observándose inversamente que durante EN los individuos menores de dicha talla tienen un mayor peso corporal, comparado con los del período LN (Fig.2). Se observaron diferencias significativas entre las regresiones para ambos períodos $\left(\mathrm{F}_{1,643}=156,65 ; P<0,05\right)$.

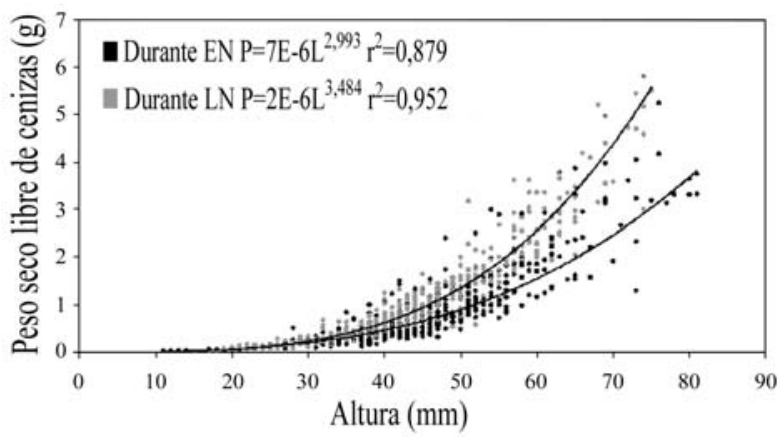

Figura 2

Diferencias en la relación talla-peso de Argopecten purpuratus en Bahía Independencia, durante los eventos EN y LN

Differences in the relation size-weight of Argopecten purpuratus at Independencia Bay, during EN and LN events

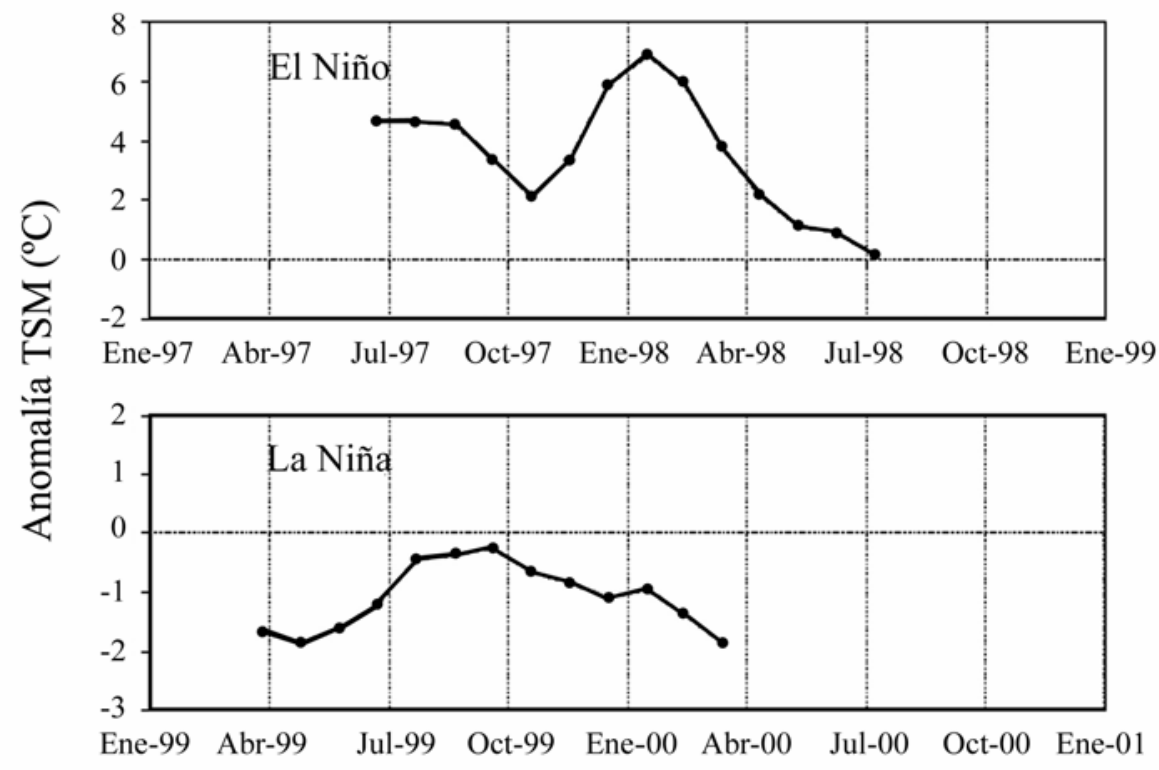

Figura 1

Anomalías de la temperatura superficial del mar (TSM), en Bahía Independencia, durante los eventos EN y LN

Anomalies of sea surface temperature at Independencia Bay, during the EN and LN events 
Tabla 1

Comparación de los parámetros de crecimiento y mortalidad de Argopecten purpuratus, en los bancos naturales de Bahía Independencia (B. I.) y Bahía Paracas (B. P.), durante los eventos EN y LN. Sin datos (s.d.)

Comparison of growth and mortality parameters of Argopecten purpuratus, in the natural beds at Independencia Bay (B. I.) and Paracas Bay (B. P.), during EN and LN events. No data (s.d.)

El Niño

\begin{tabular}{lccccclll}
\hline \multicolumn{1}{c}{ Período } & $\mathbf{H}_{\infty}$ & $\mathbf{K}$ & $\mathbf{\Phi}$ & $\mathbf{M}$ & $\mathbf{Z}$ & \multicolumn{1}{c}{ Localidad } & & \multicolumn{1}{c}{ Referencia } \\
\cline { 1 - 5 } abr 83-jun 84 & 111,5 & 2,10 & 4,42 & s.d. & s.d. & P. Atenas (B. P.) & & Wolff (1985) \\
may 83-sep 83 & 105,4 & 2,46 & 4,44 & s.d. & s.d. & P. Atenas (B. P.) & & Yamashiro \& Mendo (1988) \\
sep 87-mar 88 & 110,0 & 0,57 & 3,83 & s.d. & s.d. & La Tunga (B. I.) & & Mendo \& Jurado (1993) \\
ene 83-may 84 & 110,0 & 1,26 & 4,18 & 0,60 & 2,50 & B. Independencia & & Wolff (1987) \\
jun 97-jul 98 & 107,5 & 1,30 & 4,24 & 1,25 & 4,19 & La Pampa (B. I.) & & Este estudio \\
\hline
\end{tabular}

La Niña

\begin{tabular}{|c|c|c|c|c|c|c|c|}
\hline Período & $\mathbf{H}_{\infty}$ & $\mathbf{K}$ & $\Phi$ & $\mathbf{M}$ & $\mathbf{Z}$ & Localidad & Referencia \\
\hline 1981 & 136,0 & 0,41 & 3,88 & s.d. & s.d. & B. Independencia & Wolf (1981) \\
\hline may 84 -ago 86 & 107,6 & 0,41 & 3,67 & s.d. & s.d. & B. Independencia & Yamashiro \& Mendo (1988) \\
\hline may 84 -oct 85 & 106,1 & 0,43 & 3,68 & s.d. & s.d. & La Tunga (B. I.) & Yamashiro \& Mendo (1988) \\
\hline may 85 -abr 86 & 109,1 & 0,52 & 3,79 & s.d. & s.d. & La Pampa (B. I.) & Yamashiro \& Mendo (1988) \\
\hline mar 99-mar 00 & 118,7 & 0,50 & 3,85 & 0,56 & 2,54 & La Pampa (B. I.) & Este estudio \\
\hline
\end{tabular}

\section{Crecimiento}

La Tabla 1 muestra los valores de los parámetros de crecimiento de von Bertalanffy y el rendimiento del crecimiento durante los períodos El Niño 1997-1998 (EN) y La Niña 1999-2000 (LN), así como los estimados por otros autores en el área durante ambos eventos. Según estos valores, la tasa de crecimiento fue casi tres veces mayor en el período $\mathrm{EN}\left(\mathrm{K}=1,3\right.$ año $\left.^{-1}\right)$ si se compara con el período $\mathrm{LN}\left(\mathrm{K}=0,5 \mathrm{año}^{-1}\right)$ (Fig. 3). La altura asintótica de la conchilla durante el período $\mathrm{LN}$ fue mayor $\left(\mathrm{H}_{\infty}=118,7 \mathrm{~mm}\right)$ que durante el período $\mathrm{EN}\left(\mathrm{H}_{\infty}=107,5 \mathrm{~mm}\right)$. El valor de $\mathrm{t}_{0}$ durante el período $\mathrm{EN}$ fue de $-0,10 \mathrm{y}$ durante $\mathrm{LN}$ de $-0,43$. La edad máxima fue estimada en 1,1 y 2 años para EN y $\mathrm{LN}$, respectivamente. Asimismo, los índices de rendimiento del crecimiento, tanto $\Phi^{\prime}$ como para $\psi$, demostraron un mayor valor durante EN.

\section{Mortalidad}

La mortalidad total (Z) estimada con la curva de captura basada en la composición por tallas fue 4,19 y 2,54 para los períodos $\mathrm{EN}$ y $\mathrm{LN}$, respectivamente, mientras que la obtenida con el método de Jones \& van
Zalinge (1981) fue de 4,7 y 4,6 respectivamente. En ambos casos, la tasa de mortalidad fue más alta durante el período EN que durante LN.

Por otro lado, los valores de la tasa de mortalidad natural M obtenidos por el modelo de Hoenig (1983) fueron de 3,92 y 2,14 año $^{-1}$ para EN y LN respectivamente. Por el método de Brey (1995) se obtuvieron valores de $\mathrm{M}$ de 3,52 y 1,79 $\mathrm{años}^{-1}$, demostrando que $\mathrm{M}$ fue alrededor de dos veces mayor durante EN, en comparación con LN. Estos resultados son mostrados y comparados con otros estudios en la Tabla 1.

\section{Producción somática}

Tanto durante el período EN como LN, la población de A. purpuratus mostró una distribución de frecuencia de tallas de tipo unimodal (Fig.5). La mayor producción somática poblacional fue aportada por los grupos de talla comprendidos entre 35 a $60 \mathrm{~mm}$, durante el período EN; y entre 40 a $65 \mathrm{~mm}$, durante el período LN (Fig. 5). 


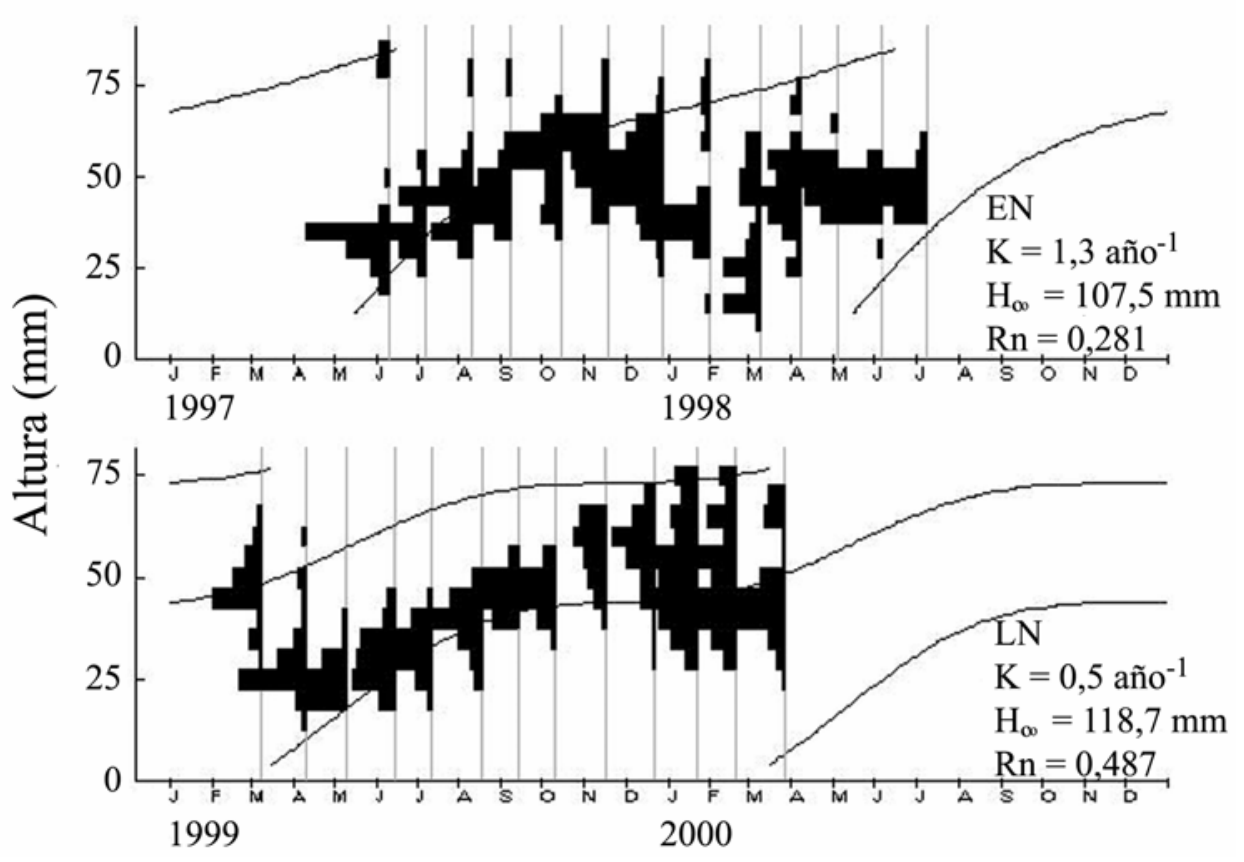

Figura 3

Estimación de la curva de crecimiento según VBFG de Argopecten purpuratus, utilizando la rutina FISAT, para los eventos EN y LN

Growth curve estimates according to VBGF model from Argopecten purpuratus, using FISAT, for EN and LN events

Los valores promedio de abundancia y biomasa durante el período EN fueron de 43,3 individuos $\mathrm{m}^{-2} \mathrm{y}$ $36,24 \mathrm{~g} \mathrm{~m}^{-2}$; mientras que durante $\mathrm{LN}$ fueron de 10,7 individuos $\mathrm{m}^{-2}$ y $15,53 \mathrm{~g} \mathrm{~m}^{-2}$.

La curva de producción somática individual durante

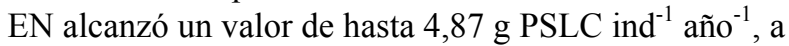
los $70 \mathrm{~mm}$ de altura; mientras que durante LN alcanzó valores de hasta $7,27 \mathrm{~g}$ PSLC ind ${ }^{-1}$ año $^{-1}$, a los $85 \mathrm{~mm}$ de altura (Fig. 4). Además se observó que en los individuos de tallas menores de $45 \mathrm{~mm}$, la mayor producción somática individual ocurrió durante EN; mientras que en las tallas mayores de $45 \mathrm{~mm}$ ocurrió durante LN (Fig. 4). Durante EN, la producción somática poblacional fue de $146,59 \mathrm{~g}$ PSLC m$^{-2}$ año $^{-1}$, mientras que durante LN disminuyó a 34,22 g PSLC $\mathrm{m}^{-2}$ año ${ }^{-1}$. La relación $\mathrm{P} / \mathrm{B}$ fue de 4,04 durante el período EN y de 2,20 durante LN, resultando casi dos veces mayor durante EN.

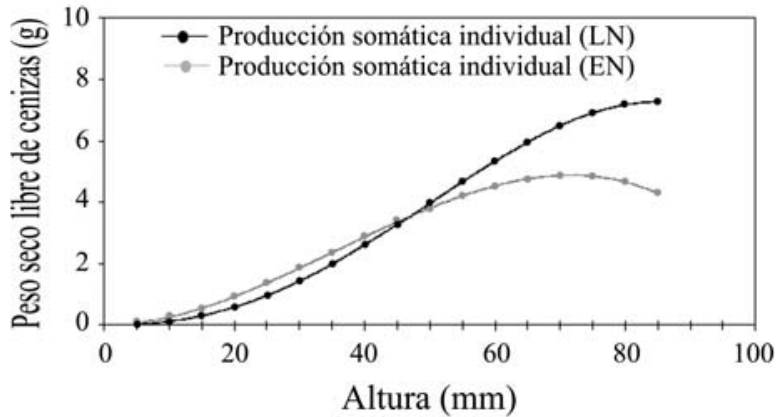

Figura 4

Diferencias en la producción somática individual de Argopecten purpuratus en Bahía Independencia, durante los eventos EN y LN

Differences in the individual somatic production of Argopecten purpuratus at Independencia Bay, during $\mathrm{EN}$ and $\mathrm{LN}$ events 


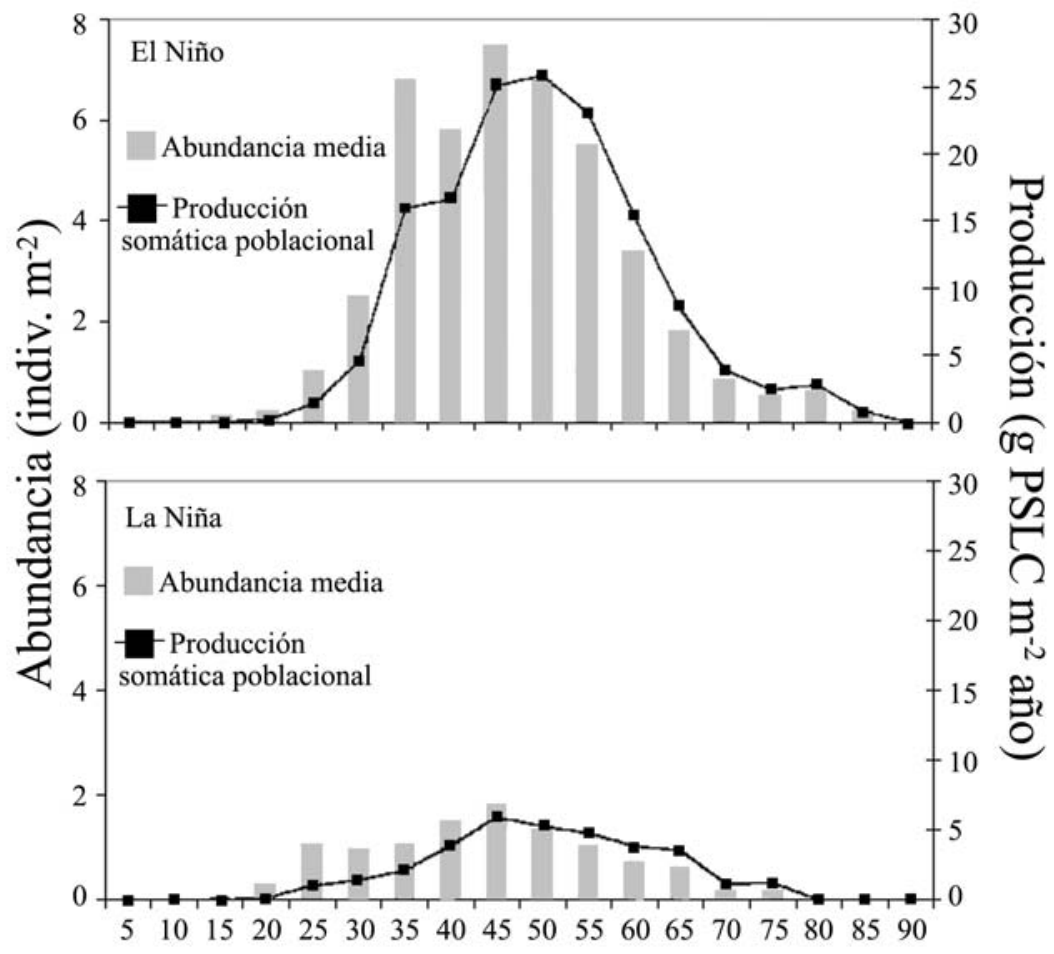

Figura 5

Diferencias de la producción somática poblacional de Argopecten purpuratus en Bahía Independencia, Pisco, durante los eventos EN y LN

Differences in the population somatic production of Argopecten purpuratus at Independencia Bay, Pisco, during EN and LN events

\section{Discusión}

Diversos autores han reportado incrementos en tamaño poblacional y en los desembarques de A. purpuratus durante y después de un evento EN (Arntz 1984, Wolff 1985, Yamashiro et al. 2002). Estos cambios han sido atribuidos a un incremento del reclutamiento, crecimiento y tasa de supervivencia como reacción al evento EN (Wolff \& Mendo 2000). Sin embargo, la información existente sobre el comportamiento de los parámetros de crecimiento y mortalidad durante los eventos cálidos EN y fríos LN no sustentan claramente una tendencia en los mecanismos antes mencionados (Tabla 1), probablemente porque no estuvieron diseñados para esclarecer dichas interrogantes aunque coincidieron con períodos cálidos o fríos. En el presente estudio se seleccionó un período cálido EN y uno frío $\mathrm{LN}$, en los cuales hubo una anomalía sostenidamente positiva o negativa respectivamente.
Urban (1994) comparó la tolerancia a temperaturas altas de 10 especies de bivalvos de Perú y Chile en relación a los eventos $\mathrm{EN}$, concluyendo que durante eventos EN débiles y fuertes, con incrementos de la temperatura de cerca de $5^{\circ} \mathrm{C}$, primaron sólo efectos subletales, debido a que las especies están adaptadas a esas temperaturas.

Se han realizado diversas estimaciones de los parámetros de crecimiento de las poblaciones naturales de A. purpuratus en el área de Pisco. Sin embargo, la diversidad metodológica y los criterios para la selección de las localidades y tiempo del estudio hace poco comparable la información. En nuestro caso, se obtuvo una tasa de crecimiento alta durante el evento EN, similar a la obtenida por otros autores (Yamashiro \& Mendo 1988). Así, Wolff $(1985,1987)$ encontró una 
Tabla 2

Comparación de los parámetros de la producción secundaria de Argopecten purpuratus con otros bivalvos del Pacífico sudeste

Parameters of secondary production from Argopecten purpuratus in comparison with other bivalves from the South Eastern Pacific

\begin{tabular}{|c|c|c|c|c|c|}
\hline Especie & $\begin{array}{c}\text { B } \\
\left(\text { gPSLC m}^{-2}\right)\end{array}$ & $\begin{array}{c}\text { Psom } \\
\left(\text { gPSLC } \mathrm{m}^{-2} \mathrm{a}^{-1}\right)\end{array}$ & $\begin{array}{l}\mathbf{P} / \mathbf{B} \\
\left(\mathbf{a}^{-1}\right)\end{array}$ & Período & Referencia \\
\hline Gari solida & 2,69 & 27,60 & 0,33 & 1991-92 & Urban \& Campos (1994) \\
\hline G. solida & 189,30 & 107,90 & 0,57 & 1990 & Urban \& Tarazona (1996) \\
\hline G. solida & 71,60 & 21,30 & 0,30 & 1992 & Urban \& Tarazona (1996) \\
\hline G. solida & 23,50 & 14,00 & 0,60 & 1993 & Urban \& Tarazona (1996) \\
\hline G. solida & 14,40 & 8,60 & 0,60 & 1994 & Urban \& Tarazona (1996) \\
\hline Protothaca thaca & 2,30 & 16,90 & 0,27 & 1991-92 & Urban \& Campos (1994) \\
\hline Semele solida & 1,91 & 4,80 & 0,19 & 1991-93 & Urban \& Campos (1994) \\
\hline Tagelus dombeii & 26,70 & 7,80 & 0,29 & $1991-94$ & Urban (1996) \\
\hline Venus antiqua & 69,56 & 40,69 & 0,59 & 1994 & Clasing et al. (1994) \\
\hline V. antiqua & 122,00 & 22,00 & 0,18 & $1991-92$ & Urban (1996) \\
\hline Ensis macha & 43,60 & 9,70 & 0,22 & $1991-92$ & Urban (1996) \\
\hline Argopecten purpuratus & 36,24 & 146,59 & 4,04 & $1997-98$ & Este estudio \\
\hline A. purpuratus & 15,53 & 34,22 & 2,20 & $1999-2000$ & Este estudio \\
\hline
\end{tabular}

alta tasa de crecimiento y un incremento del desove para A. purpuratus durante el ENOS 1982-83, explicando el efecto positivo de este evento por el hecho de que esta especie es un relicto de una fauna subtropical del Mioceno. Por eso las temperaturas altas durante el ENOS tendrían efecto positivo sobre el crecimiento y reproducción de la especie.

Mendo \& Jurado (1993) reportan una tasa de crecimiento de 0,53 para el EN moderado 1987-88 en los bancos naturales, con mayores tasas de crecimiento en sistemas de cultivo suspendido $(K=2,9)$. Mientras que Wolff (1981) y Yamashiro \& Mendo (1988) reportan, para períodos fríos, menores tasas de crecimiento, con valores entre 0,41 a 0,52 , similar al obtenido en este estudio para el período 1999-2000, pero menores a los estimados bajo condiciones EN. Por lo tanto, es evidente un efecto $\mathrm{EN}$ al incrementar el crecimiento individual de la especie.

Yamashiro et al. (2002) señalan que la actividad extractiva de concha de abanico durante el EN 1997-98 se sustentó en los constantes reclutamientos producidos en Bahía Independencia, asociados a un proceso reproductivo continuo; sin embargo, los desembarques y CPUE fueron disminuyendo paulatinamente, debido a la intensa extracción de tallas menores a las comerciales, que afectaron las tasas de renovación poblacional del recurso. En el 2000, la explotación de concha de abanico mostró una tendencia decreciente de los desembarques mensuales, con un total anual de $4023 \mathrm{t}$.

En la zona de Pisco, la extracción de invertebrados viene registrando un alto porcentaje de ejemplares por debajo de la talla mínima permisible en los últimos años, como lo señalan Wolff \& Mendo (2000). Estos mismos autores postulan que bajo un escenario de cambio de la normalidad a condiciones de EN, las metapoblaciones de concha de abanico reaccionan a través de un incremento del reclutamiento, crecimiento $\mathrm{y}$ tasa de supervivencia en los bancos naturales, y proponen un modelo para optimizar los rendimientos anuales, considerando factores ecológicos y económicos. Mendo \& Wolff (2002) señalan que los valores acumulados de los desembarques registrados cuatro años después del inicio de un evento $\mathrm{EN}$ fuerte, muestran que EN 1982-83 fue más productivo que EN 
1997-98. Mendo \& Wolff (2003) mencionan también que la producción de concha de abanico (en términos de desembarque) es alterada positivamente sólo con la ocurrencia de EN fuertes como los de 1983 y 1998. Asimismo, podría asumirse que A. purpuratus tiene una estrategia reproductiva oportunista que la lleva a una inversión de energía en la producción de gametos cuando las condiciones medio ambientales son favorables. Esta conclusión es concordante con el efecto ENSO sobre el crecimiento, el cual fue más rápido durante $\mathrm{EN} \mathrm{97/98} \mathrm{que} \mathrm{durante} \mathrm{LN} \mathrm{99/00} \mathrm{(Tabla}$ 1) y sobre la gametogénesis, la que fue ampliamente favorecida (Illanes et al. 1985, Ishiyama \& Shiga 1997). No obstante, la evidencia empírica indica que elevadas temperaturas limitan la eficiencia del crecimiento en los pectínidos y que la adaptación no compensa totalmente este efecto (Heilmayer et al. 2004).

Considerando la relación talla-peso seco libre de cenizas (PSLC) que se da en ambos eventos, se observa que las tallas menores de 25,5 $\mathrm{mm}$ durante EN tuvieron un mayor peso somático que durante $\mathrm{LN}$ debido a un crecimiento más acelerado de estas tallas, que tendieron a una mayor acumulación de tejido somático. Mientras que para tallas mayores de $25 \mathrm{~mm}$ (coincidente con la talla de primera madurez; C. Yamashiro, com. pers.) durante EN presentaron pesos individuales menores que en LN, debido a que probablemente la especie invirtió más energía de su metabolismo en la producción de gametos y por eso incrementó la intensidad en la frecuencia de maduración y desove, con lo cual disminuyó la masa de tejido somático. En este caso, un menor peso individual estaría más relacionado con una alta tasa reproductiva, que con otros factores bióticos como disponibilidad de alimento, competencia, etc. Esta hipótesis quedó también evidenciada con las curvas de producción somática individual donde se observa claramente un descenso de la producción somática individual para tallas mayores de $45 \mathrm{~mm}$, que son las que ya iniciaron el proceso reproductivo y tendrían una alta tasa reproductiva. Inversamente, durante el periodo $\mathrm{LN}$, para tallas mayores de $45 \mathrm{~mm}$, los individuos tuvieron mayor masa corporal, lo que sería explicado porque la especie tiene una menor tasa reproductiva y una mayor producción somática, con lo cual no encuentra condiciones ambientales que estimulen en gran medida la producción gonádica.
Al respecto, Barber \& Blake (1983) señalan que las poblaciones de Argopecten irradians en Florida, EUA, límite sur de la distribución de la especie, muestran una tasa metabólica incrementada, asociada con una temperatura más alta. Esto, junto con un menor abastecimiento de alimento, hace que las conchas de abanico tengan menos energía disponible para la reproducción, limitando su distribución hacia el sur. MacDonald \& Thompson (1986), al estudiar dos poblaciones no explotadas de Placopecten magellanicus de Terranova, encontraron que una población (Colinet) tenía una tasa de crecimiento lento, una baja producción reproductiva y una baja relación $\mathrm{P} / \mathrm{B}$, en comparación con la otra población (Sunnyside). En Colinet, las clases de edad jóvenes estuvieron bien representadas y fueron las que más aportaron a la producción, siendo el $46 \%$ exportado como gametos. En Sunnyside dominaron las clases de mayor edad y destinando el $72 \%$ de la producción a la reproducción. En nuestro caso, la población de A. purpuratus que presenta una mayor tasa de crecimiento y mayor tasa reproductiva estaría asociada con un mayor P/B.

Respecto a la relación entre masa corporal y la tasa de renovación anual $\mathrm{P} / \mathrm{B}$, se observa que una menor tasa $\mathrm{P} / \mathrm{B}$ durante La Niña está asociada con una mayor masa corporal individual, una mayor edad máxima (crecimiento más lento) y una menor tasa de mortalidad total. Inversamente, durante el periodo El Niño, una mayor tasa $\mathrm{P} / \mathrm{B}$ estaría involucrada con un menor peso corporal individual, una menor edad máxima y una mayor tasa de mortalidad total. Esta relación fue observada en el venérido Eurhomalea exalbida en Patagonia (Lomovasky et al. 2002).

La tasa $\mathrm{P} / \mathrm{B}$ es una medida estandarizada que permite comparaciones entre especies que tienen diferentes biomasas individuales y es calculada en este estudio por el método de la tasa de crecimiento específico en peso. Los valores de $\mathrm{P} / \mathrm{B}$ de esta especie son mayores comparados a datos reportados de otras familias de bivalvos del Pacífico Sudeste; así Urban \& Campos (1994) reportaron un P/B para Gari solida, Semele solida y Protothaca thaca, de 0,334; 0,188 y 0,269 respectivamente; Clasing et al. (1994) determinaron para Venus antiqua un valor de $\mathrm{P} / \mathrm{B}$ de 0,585; Urban (1996) registró para $V$. antiqua, Tagelus dombeii y Ensis macha, valores de 0,18, 0,29 y 0,22 respectivamente; Terry \& Mendo (2002) reportaron para Aulacomya ater un estimado de 0,78. Stotz \& 
González (1997) calcularon para esta especie, en la bahía de Tongoy, una relación P/B de 2,08 y una tasa de crecimiento más rápida que los estimados anteriores, tomando 18 meses en alcanzar la talla comercial $(>90$ $\mathrm{mm})$.

El presente trabajo comparó por primera vez la producción secundaria de la especie durante EN y LN, demostrando que la tasa de crecimiento durante EN 1997-98 fue casi tres veces mayor que durante LN 1999-2000. El estudio demuestra también que el efecto positivo de EN sobre la producción es tanto a nivel de los individuos como de la población de A. purpuratus. Asimismo, estos parámetros de crecimiento y producción calculados podrían ser usados como índices en la construcción de modelos ecotróficos de los principales recursos pesqueros en la costa peruana, simulando diferentes situaciones ambientales durante EN y LN.

\section{Agradecimientos}

Al Instituto Alfred Wegener para Estudios Marinos y Polares (Alemania) y a la Universidad Nacional Mayor de San Marcos, que brindaron el apoyo financiero para el desarrollo de la investigación a través de los diversos proyectos ejecutados. También a los miembros del grupo de investigación DePSEA, Laboratorio de Ecología Marina, Universidad San Marcos, quienes colaboraron en la toma de muestras biológicas y el análisis de datos. Se agradece de manera especial a los tres evaluadores anónimos por la crítica invaluable y la revisión del manuscrito.

\section{Literatura citada}

Arntz WE. 1984. El Niño and Peru: positive aspects. Oceanus 27: 36-39.

Arntz WE. 1986. The two faces of El Niño 1982-1983. Meeresforschung 31: 1-46.

Arntz WE \& E Fahrbach. 1996. El Niño: Experimento climático de la naturaleza. 312 pp. Fondo de Cultura Económica, México.

Arntz WE \& J Tarazona. 1990. Effects of El Niño 19821983 on benthos, fish and fisheries off the South American Pacific Coast. En: Glynn PW (ed). Global Ecological consequences of the 1982-83 El NiñoSouthern Oscillation. Elsevier Oceanography Series 52: 323-360. Elsevier, Amsterdam.

Barber B \& N Blake. 1983. Growth and reproduction of the bay scallop, Argopecten irradians (Lamarck) at its southern distributional limit. Journal of Experimental Marine Biology and Ecology 36(3): 247-256.

Brey T. 1995. Empirische Untersuchungen zur Populationsdynamick makrobenthischer Evertebraten. Habilitation Thesis, University of Bremen, Germany, 161 pp.

Brey T. 2001. Population dynamics in benthic invertebrates. A virtual handbook. http://awibremerhaven.de/Benthic/ Ecosystem / FoodWeb / Handbook/main.html. Alfred Wegener Institute for Polar and Marine Research, Germany.

Clasing E, T Brey, R Stead, J Navarro \& G Ascencio. 1994. Population dynamics of Venus antiqua (Bivalvia: Veneracea) in the Bahía de Yaldad, Isla de Chiloé, southern Chile. Journal of Experimental Marine Biology and Ecology 177: 171-186.

Crisp D. 1984. Energy flow measurements. En: Holme N \& A McIntyre (eds). Methods for the study of marine benthos, pp. 284-372. Blackwell, London.

Gayanilo FC, P Sparre \& D Pauly. 1995. The FAOICLAM Stock Assessment Tools (FISAT) User's Guide. FAO Computerized Information, Series Fisheries 6: 1126.

Heilmayer O, T Brey \& HO Portner. 2004. Growth efficiency and temperature in scallops: a comparative analysis of species adapted to different temperatures. Functional Ecology 18: 641-647.

Hoenig JM. 1983. Empirical use of longevity data to estimate mortality rates. Fishery Bulletin 82: 898-903.

Illanes JE, S Akaboshi \& E Uribe. 1985. Efectos de la temperatura en la reproducción del ostión del norte (Argopecten purpuratus) en la Bahía Tongoy durante el fenómeno El Niño 1982-83. Investigación Pesquera 32: 167-173.

Ishiyama V \& B Shiga. 1997. El fenómeno El Niño 199293: su influencia en la reproducción de la concha de abanico Argopecten purpuratus. Revista Científica Wiñay Yachay (Perú) 1(2): 41-46.

Jones R \& NP van Zalinge. 1981. Estimations of mortality rate and population size for shrimp in Kuwait waters. Kuwait Bulletin of Marine Science 2: 273-288.

Lomovasky B, T Brey, E Morriconi \& J Calvo. 2002. Growth and production of the venerid bivalve Eurhomalea exalbida in the beagle Channel, Tierra del Fuego. Journal of Sea Research 48: 209-216.

Macdonald B \& R Thompson. 1986. Production, dynamics and energy partitioning in two populations of the giant scallop Placopecten magellanicus. Journal of Experimental Marine Biology and Ecology 101(3): 285299. 
Mendo J \& E Jurado. 1993. Length-based growth parameter estimates of the Peruvian scallop (Argopecten purpuratus). Fisheries Research 15(4): 357-367.

Mendo J \& M Wolff. 2002. Pesquería y manejo de la concha de abanico (Argopecten purpuratus) en la Bahía Independencia. En: Mendo J \& M Wolff (eds). Memorias de la I Jornada Científica Bases ecológicas y socioeconómicas para el manejo de los recursos vivos de la reserva nacional de Paracas, pp. 188-194. Universidad Nacional Agraria La Molina, Lima.

Mendo J \& M Wolff. 2003. El impacto de El Niño sobre la producción de concha de abanico (Argopecten purpuratus) en Bahía Independencia, Pisco, Perú. Ecología Aplicada 2(1): 51-57.

Pauly D. 1980. On the interrelationships between natural mortality, growth parameters, and mean environmental temperature in 175 fish stocks. ICES Journal of Marine Science 39(2): 175-192.

Pauly D. 1983. Algunos métodos simples para la evaluación de recursos pesqueros tropicales. FAO Documento Técnico de Pesca 234: 1-49.

Pauly D \& JL Munro. 1984. Once more on growth comparison in fish and invertebrates. Fishbyte 2: 21 .

Sokal RR \& FJ Rholf. 1981. Biometry, $2^{\text {nd }}$ edition, 859 pp. WH Freeman and Company, San Francisco.

Stotz W \& S González. 1997. Abundance, growth, and production of the sea scallop Argopecten purpuratus (Lamarck 1819): bases for sustainable exploitation of natural scallop beds in north-central Chile. Fisheries Research 32: 173-183.

Tarazona J \& W Arntz. 2001. The Peruvian Coastal Upwelling System. En: Seeliger U \& B Kjerfve (eds) Coastal Marine Ecosystems of Latin America. Ecological Studies 144: 229-244. Springer Verlag, Berlín.

Terry C \& J Mendo. 2002. Crecimiento, mortalidad y producción del choro (Aulacomya ater) en Bahía Independencia durante el período 1999-2000. En: Mendo J \& M Wolff (eds) Memorias I Jornada Científica Bases ecológicas y socioeconómicas para el manejo de los recursos vivos de la Reserva Nacional de Paracas, pp. 95102. Universidad Nacional Agraria La Molina, Lima.
Urban HJ. 1994. Upper temperature tolerance of ten bivalve species of Peru and Chile related to El Niño. Marine Ecology Progress Series 107(1): 139-145.

Urban HJ. 1996. Population dynamics of the bivalves Venus antiqua, Tagelus dombeii, and Ensis macha from Chile at $36^{\circ}$ S. Journal of Shellfish Research 15(3): 719-727.

Urban HJ \& B Campos. 1994. Population dynamics of the bivalves Gari solida, Semele solida and Protothaca thaca from a small bay in Chile at $36^{\circ} \mathrm{S}$. Marine Ecology Progress Series 115: 93-102.

Wolff M. 1981. Die Nutzung von Muscheln an der Südküste Perus. Diplomarbeit IFM/CA, 110 pp. Universität zu Kiel, Kiel.

Wolff M. 1985. Abundancia masiva y crecimiento de preadultos de la concha de abanico peruana (Argopecten purpuratus) en la zona de Pisco, bajo condiciones El Niño 1983. En: Arntz W, A Landa \& J Tarazona (eds). El Niño: su impacto en la fauna marina, pp. 87-89. Volumen Extraordinario. Boletín del Instituto del Mar del Perú, Callao, Perú.

Wolff M. 1987. Population dynamics of the Peruvian scallop Argopecten purpuratus during the El Niño phenomena of 1983. Canadian Journal of Fisheries and Aquatic Sciences 44: 1684-1691.

Wolff M \& J Mendo. 2000. Management of the Peruvian scallop (Argopecten purpuratus) metapopulation with regard to enviromental change. Aquatic Conservation: Marine and Freshwater Ecosystems 10: 117-126.

Yamashiro C \& J Mendo. 1988. Crecimiento de la concha de abanico (Argopecten purpuratus) en la Bahía Independencia, Pisco - Perú. En: Salzwedel H \& A Landa (eds), Recursos y dinámica del ecosistema de afloramiento, pp. 163-168. Volumen Extraordinario. Boletín del Instituto del Mar del Perú, Callao, Perú.

Yamashiro C, J Rubio, A Taipe \& S Aguilar. 2002. Fluctuaciones de la población de concha de abanico Argopecten purpuratus (Lamarck, 1819) en la Bahía Independencia (Pisco, Perú) durante el período 19842000. En: Mendo J \& M Wolff (eds), Memorias de la I Jornada Científica Bases ecológicas y socioeconómicas para el manejo de los recursos vivos de la reserva nacional de Paracas, pp. 77-87. Universidad Nacional Agraria La Molina, Lima. 